OPEN ACCESS

Edited by:

Frederic Lamoth,

Centre Hospitalier Universitaire Vaudois (CHUV), Switzerland

Reviewed by:

Dionysios Neofytos,

Hôpitaux Universitaires de Genève

(HUG), Switzerland

Olga Matos,

Universidade Nova de Lisboa,

Portugal

*Correspondence:

Catherine Cordonnier

catherine.cordonnier@aphp.fr

Specialty section

This article was submitted to

Infectious Diseases,

a section of the journal

Frontiers in Microbiology

Received: 15 February 2017 Accepted: 05 April 2017 Published: 24 April 2017

Citation:

Robin C, Alanio A, Gits-Muselli M, la Martire G, Schlemmer F, Botterel F, Angebault $C$, Leclerc $M$, Beckerich $F$

Redjoul R, Pautas C, Toma A,

Maury S, Bretagne S and

Cordonnier C (2017) Molecular

Demonstration of a Pneumocystis

Outbreak in Stem Cell Transplant Patients: Evidence for Transmission in

the Daycare Center.

Front. Microbiol. 8:700

doi: $10.3389 /$ fmicb.2017.00700

\section{Molecular Demonstration of a Pneumocystis Outbreak in Stem Cell Transplant Patients: Evidence for Transmission in the Daycare Center}

\author{
Christine Robin 1,2, Alexandre Alanio 3, 4, 5, Maud Gits-Muselli ${ }^{3,4}$, Giulia la Martire ${ }^{1}$, \\ Frédéric Schlemmer 2,6, Françoise Botterel ${ }^{2,7}$, Cécile Angebault ${ }^{2,7}$, Mathieu Leclerc ${ }^{1,2}$, \\ Florence Beckerich ${ }^{1,2}$, Rabah Redjoul ${ }^{1,2}$, Cécile Pautas ${ }^{1}$, Andrea Toma ${ }^{1}$, \\ Sébastien Maury ${ }^{1,2}$, Stéphane Bretagne ${ }^{3,4,5}$ and Catherine Cordonnier ${ }^{1,2 *}$ \\ 1 Department of Hematology, Assistance Publique-Hôpitaux de Paris, Henri Mondor Teaching Hospital, Créteil, France, \\ ${ }^{2}$ Paris-Est Créteil University, Créteil, France, ${ }^{3}$ Parasitology-Mycology Laboratory, Assistance Publique-Hôpitaux de Paris, \\ Lariboisière Saint-Louis Fernand Widal Hospital, Paris, France, ${ }^{4}$ Paris-Diderot, Sorbonne Paris Cité University, Paris, France, \\ ${ }^{5}$ Molecular Mycology Unit, National Reference Center of Invasive Mycosis and Antifungals, Centre National de la Recherche \\ Scientifique, Institut Pasteur, URA3012, Paris, France, ${ }^{6}$ Unit of Pneumology, Intensive Care Department, Assistance \\ Publique-Hôpitaux de Paris, Henri Mondor Teaching Hospital, DHU A-TVB, Créteil, France, ${ }^{7}$ Parasitology-Mycology \\ Laboratory, Assistance Publique-Hôpitaux de Paris, Henri Mondor Teaching Hospital, Créteil, France
}

Pneumocystis jirovecii pneumonia (PCP) is a life-threatening infection in hematology. Although occasionally reported, the role of interhuman transmission of $P$. jirovecii in PCP, compared to that of reactivation, remains an unresolved question; the recommendation to isolate PCP patients in the hematology ward are not well evidence-based. Following an unexpected increase in the number of febrile pneumonia patients with $P$. jirovecii DNA detected in respiratory samples in our hematology ward, we explored 12 consecutive patients from November 2015 to May 2016. Genotyping of $P$ jirovecii was performed using microsatellite markers. The frequency of simultaneous occupancy of these 12 patients in the same unit on the same day from 4 months prior to the first diagnosis was recorded. In three patients, the P. jirovecii genotype could not be determined because DNA was insufficient. One rare single genotype (Gt2) was found in four of the other nine, all allogeneic stem cell transplant recipients. The transmission map showed that these 4 patients had multiple opportunities to meet on the same day (median, 6.5; range, 4-10) at the daycare center. It was much less among the eight non-Gt2 patients (median, 1; range, $0-9 ; P=0.048)$. This study, based on modern molecular technics, strongly suggests that interhuman transmission of $P$. jirovecii between allogeneic stem cell transplant recipients is possible. P. jirovecii DNA detected in respiratory specimens supports that isolation and respiratory precautions be recommended in such cases in the hematology ward.

Keywords: Pneumocystis jirovecii, genotyping, microsatellite, short tandem repeat, nosocomial infection 


\section{INTRODUCTION}

Pneumocystis jirovecii pneumonia (PCP) is a life-threatening fungal complication in hematology patients, particularly recipients of allogeneic hematopoietic stem cell transplantation (HSCT), where mortality is greater than 50\% (Cordonnier et al., 2016). It is usually accepted that $P$. jirovecii is acquired early in life and that individuals are continuously exposed to it through the air (Gajdusek, 1957; Gigliotti and Wright, 2012; Morris and Norris, 2012). However, it is unclear whether infection is the exclusive result of recent exposure or if reactivation of the earlyacquired strain could also participate in the development of PCP. Person-to-person transmission has been documented during outbreaks, especially in solid organ transplant wards (Yiannakis and Boswell, 2016). To avoid such transmission, recent guidelines for managing PCP in hematology patients published by the European Conference on Infections in Leukemia group (Alanio et al., 2016c; Maertens et al., 2016; Maschmeyer et al., 2016) state that "it seems reasonable that severely immunocompromised patients should avoid contact with patients with documented PCP." However, because data is lacking in this population, this is a C-III recommendation (e.g., there is poor evidence to support it). Similarly, the 2007 guidelines for isolation precautions from the US Centers for Disease Control and Prevention advises that PCP-infected patients should not be placed in the same room as immunocompromised patients, and other standard precautions should be taken (Siegel et al., 2007).

Genotyping studies of outbreaks in renal (Thomas and Limper, 2004; de Boer et al., 2011; Yiannakis and Boswell, 2016) or liver (Desoubeaux et al., 2016) transplant units were of major importance when reconsidering the mechanism of PCP development and supporting interhuman or environmental transmission of $P$. jirovecii. Additionally, the presence of P. jirovecii in the exhaled air of PCP patients (Choukri et al., 2010; Damiani et al., 2012) and the common genotype found in infected patients and colonized health care workers (Damiani et al., 2012) strongly suggest that PCP infection can also be nosocomial. However, only 16 of the reported outbreaks in kidney recipients thus far (Yiannakis and Boswell, 2016) were documented using molecular biology, and no firm statement on the transmission mode has been made.

The methods for detecting and genotyping $P$. jirovecii have greatly evolved over the last years. A PCP diagnosis increasingly relies on real-time quantitative PCR ( $\mathrm{PPCR}$ ) instead of microscopy and immunofluorescence staining (Alanio et al., 2016c). The qPCR assay increases the rate of detection and provides additional clues for elucidating the source of contamination. Various methods of genotyping have been developed (reviewed in Alanio et al., 2016c); multilocus sequence typing and internal transcribed spacer (ITS) sequencing are the most common. New genotyping methods based on short tandem repeat markers located in the nuclear genome are now available, allowing easier and more rapid analysis of clustered infections and accurate detection of mixtures (Parobek et al., 2014; Alanio et al., 2016b).

To our knowledge, this is the first possible interhuman transmission of $P$. jirovecii documented using modern molecular tools in the hematology ward. Microsatellite marker genotyping was used during an outbreak of febrile pneumonia in a hematology ward, and positive $P$. jirovecii detection was employed. This study shows that hematology patients can be infected by $P$. jirovecii genotypes transmitted from other at-risk patients in the ward. This clearly supports a strong recommendation for isolation and respiratory precautions where $P$. jirovecii is detected in respiratory DNA specimens from patients cared for in the same hematology ward.

\section{MATERIALS AND METHODS}

\section{Study Setting}

The hematology department comprises 26 single-occupancy rooms for conventional hospitalization. It includes one unit (Unit 1) without air filtration that has eight beds, two units with laminar air-flow filtration that have six (Unit 2) and 12 (Unit 3) beds, and a daycare clinic, which cares for 30-50 patients daily on the upper floor. When the patients are at the daycare center, they all arrive between 8 and 9 a.m. and may stay several hours on site, sharing a unic waiting room. The department mainly recruits patients with acute leukemia, myelodysplastic syndrome, myeloproliferative disorders, and aplastic anemia. Around 40 allogeneic HSCTs are performed annually. Patients, hospital stays, and daycare center stays numbered 255,505 , and 2,450, respectively, and the median hospital stay was 20 days. These values have not appreciably varied since 2013.

Procedures for PCP prophylaxis in acute lymphoblastic leukemia and lymphoma patients and in HSCT recipients are in writing. The first-line recommended regimen is a single doublestrength tablet of trimethoprim-sulfamethoxazole (TMP-SMX) thrice weekly, but if not tolerated, the second-line is atovaquone (750 mg oral suspension twice daily) or pentamidine aerosol monthly. Patients with pneumonia, except those in palliative care, are investigated by fiberoptic bronchoscopy and bronchoalveolar lavage (BAL) with a systematic search for P.jirovecii by MayGrünwald Giemsa (MGG) staining (RAL-555; RAL Reagents, Martillac, France) and an indirect immunofluorescence assay (IFA; MONOFLUO ${ }^{\mathrm{TM}}$ kit, $P$. jirovecii; Bio-Rad, Marnes la Coquette, France) as previously described (Botterel et al., 2012). Since 2013, a qPCR targeting the mitochondrial LSU of the rRNA of $P$. jirovecii has been routinely performed in respiratory specimens (BAL fluid or upper respiratory tract samples) (Botterel et al., 2012). Other causes of pneumonia are investigated using systematic laboratory procedures to identify bacterial, viral, and other fungal pathogens as well as alveolar hemorrhage and alveolar proteinosis. A serum (1-3) $\beta$-D-glucan test (Fungitell assay, Associates of Cape Cod, Inc., Cap Cod, Massachusetts), when requested, was performed according to manufacturer recommendations and was considered positive at $>80 \mathrm{pg} / \mathrm{mL}$. However, the test was not routine at this time and could not be performed within $48 \mathrm{~h}$ of the BAL for all patients.

Figure 1 shows the frequency of febrile pneumonia and positive DNA tests for $P$. jirovecii between January 2013 and May 2016. It was 4 to 5 cases per year before the fall of 2015, but 4 cases were observed from November 6 to 14, 2015. Eight additional cases were observed from December 2015 to May 


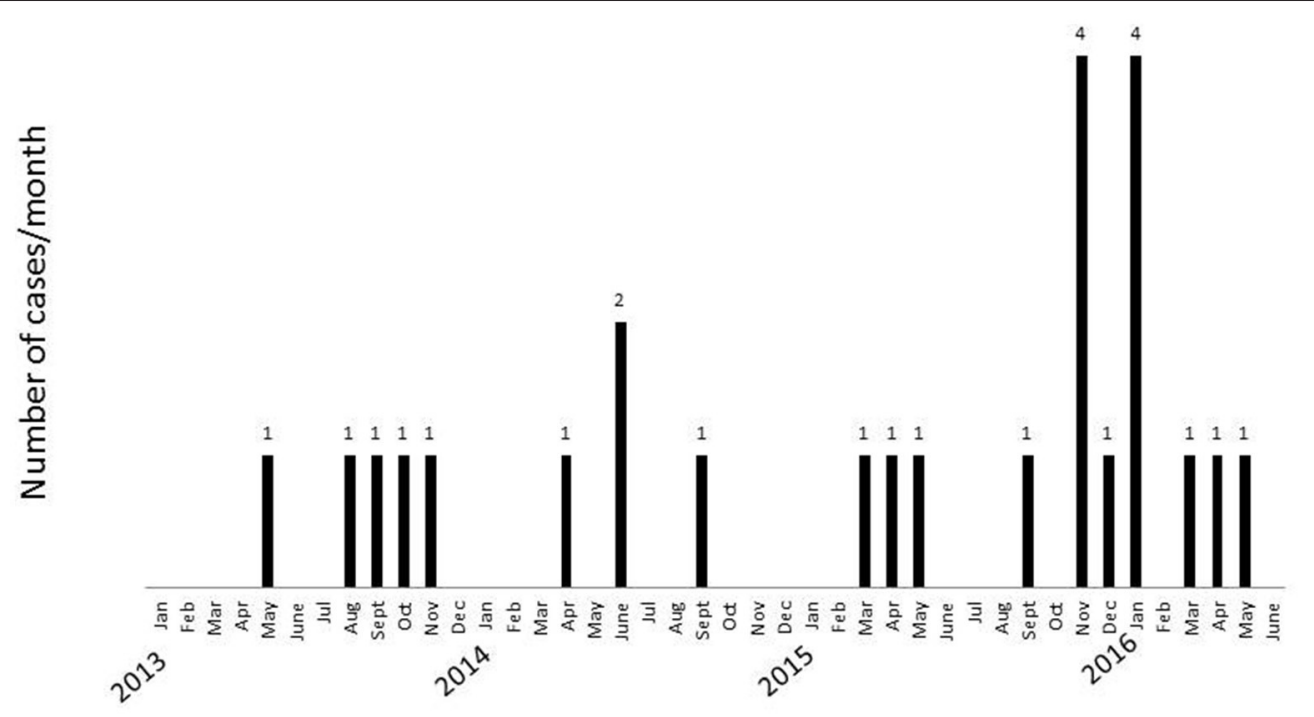

FIGURE 1 | Number of pneumocystis cases observed in the hematology department between January 2013 and May 2016.

2016. This unexpected high incidence led to a search for evidence of transmission using $P$. jirovecii genotyping and an analysis of patient presence in the ward. According to French Health Public Law (CSP Art L1121-1.1), such an investigation does not require specific informed consent or ethics committee approval.

\section{Molecular Typing}

Molecular typing of $P$. jirovecii was performed on DNA extracted from respiratory specimen (BAL or/and sputum) that were frozen at $-20^{\circ} \mathrm{C}$. Microsatellite analysis was performed according to previously published methods (Gits-Muselli et al., 2015). Briefly, tests for six nuclear microsatellite markers (\#022, $\# 108$, \#138, \#189, \#278, and \#279) were performed using fluorescent primers. The size of the PCR product obtained after capillary electrophoresis depends on the number of short tandem repeats for each marker. For a given marker, the size that is proportional to the number of repeats defines an allele. A unique combination of one specific allele per marker identifies a specific genotype. The genotypes were arbitrarily attributed numbers and were considered to be found if all alleles were unique for a given marker (pure genotype) or if more than one allele was found for one marker (multiple genotypes). Otherwise (i.e., when multiple alleles were observed for more than 1 marker), the genotype could not be determined, and the result was reported as a "mixture" of various genotypes.

\section{RESULTS \\ PCP Patients}

Twelve consecutive PCP patients hospitalized from November 2015 to May 2016 were included, and their characteristics are summarized in Table 1. All were investigated by fiberoptic bronchoscopy and BAL. Seven were allogeneic HSCT recipients. At BAL, all 12 patients were symptomatic, febrile, and hypoxemic, and lung CT images were consistent with interstitial pneumonia, mainly diffuse, patchy, ground-glass opacities. A serum (1-3) $\beta$-D-glucan test was available for nine patients and was positive for six (Patients 1, 3-6, and 10; Table 1). No serum (1-3) $\beta$-D-glucan test was performed for Patients 8, 9, and 12. At diagnosis, PCP prophylaxis was atovaquone in three patients and pentamidine aerosols in one because these patients were intolerant of TMP-SMX. Eight patients had no prophylaxis, five because of the absence of recommendations for mixed phenotype $\mathrm{AL}(n=2)$ or for $\operatorname{AML}(n=3)$ and one because of deep neutropenia after HSCT. In the other two, prophylaxis was stopped several months prior, after withdrawing immunosuppressive drugs. As a result, none of the 12 patients received TMP-SMX prophylactically.

\section{Genotyping Results}

In three patients (Patients 8, 11, and 12), the $P$. jirovecii genotype could not be determined because insufficient amounts of DNA were obtained. In Patient 1, only one marker (\#022) was amplified, but with a different allele from other patients, allowing the assumption that this patient had a specific genotype (Gt5). In the eight remaining patients, the six markers were correctly amplified. One genotype (Gt2) was found pure in four patients (Patients 2, 5, 7, and 10), all of whom were diagnosed within a timeframe of 4 months (November 9, 2015 to March 15, 2016). Of note, Gt 2 had not been found in our collection of genotyped $P$. jirovecii sspecimens from France and Europe ( $n>300$ in total) (Alanio et al., 2014, 2016a; Gits-Muselli et al., 2015). The various Gt2 alleles were searched for but not found in the other patients, confirming that they did not harbor it.

Patients 4 and 9 shared Gt3 genotype, in addition to other alleles in Patient 4, and in addition to Gt4 in Patient 9. Of note, Gt3 has been very rarely observed in our experience (one patient from Paris, one from Germany, and one from The Netherlands out of at least 300 patients). 


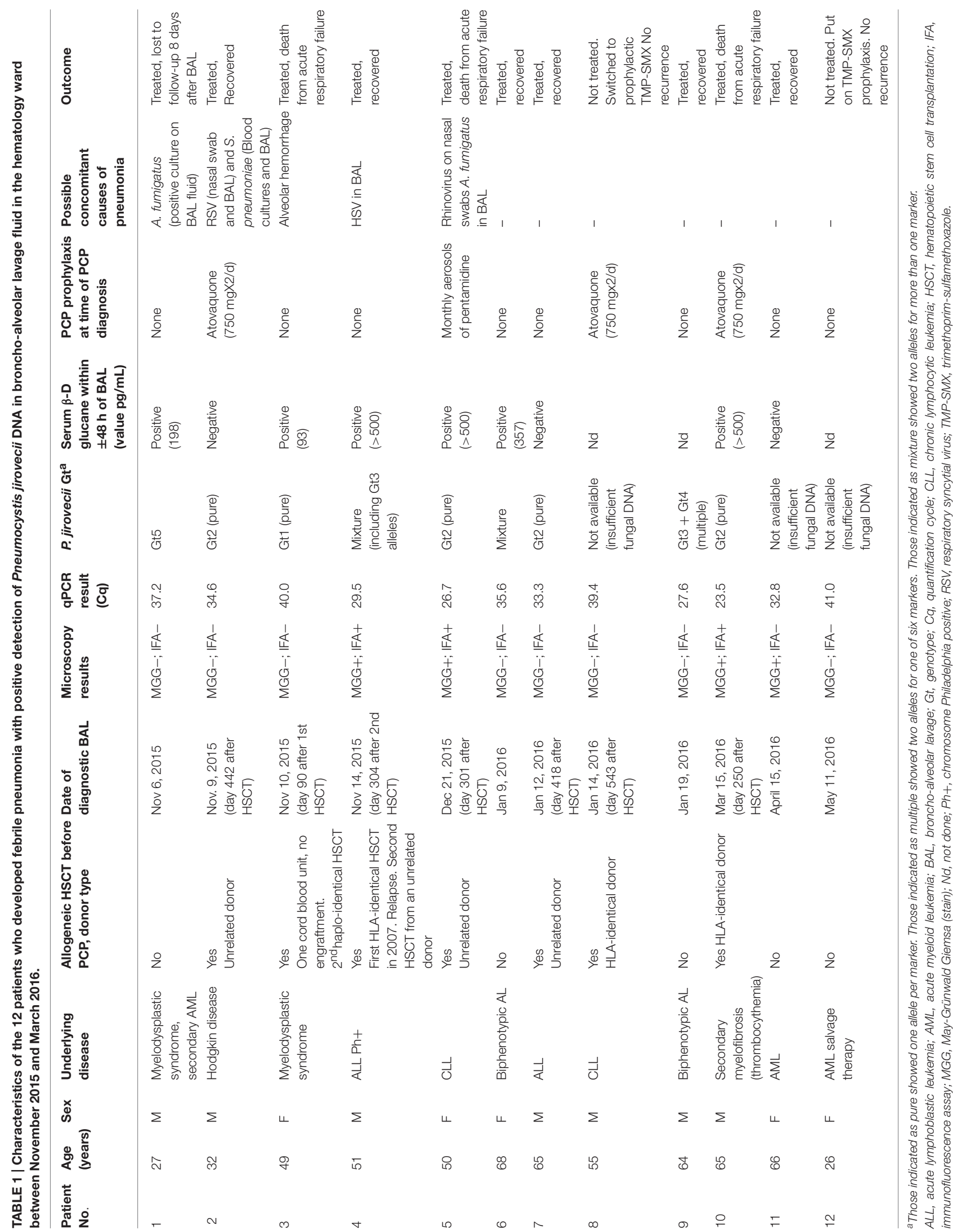




\section{Transmission Map}

Gt2 was first observed on November 9, 2015 in the BAL of Patient 2 (Figure 2). To explore a possible nosocomial transmission through close contact between the four Gt2 patients, we collected all the dates, times of stays, and meeting sites within the hospital from July 2015 to May 2016 (i.e., starting 4 months before and ending 6 months after the first case). Direct transmission was deemed possible if patients were found to be in the same room on the same day. All contacts between these four patients occurred in the daycare center, which has only one waiting room (Figure 2). The median number of contacts was 6.5 (range, 410). In contrast, the median number of contacts between the other eight patients and any Gt2 patient was significantly lower (median, 1 ; range, $0-9 ; P=0.048$ ). Additionally, non-Gt2 to nonGt2 patient contact was zero for five patients, one for one of them, and three for two of them. Figure 2 strongly suggest that either patient 2 or patient 5 was the source of Gt2 transmission.

Moreover, 1 month before diagnosis in December 2015, Patient 5 was hospitalized for fever, bronchopulmonary symptoms, and hypoxia in a different unit than Patient 2. The chest CT scan showed micronodules in the upper right lobe, indirect investigations were negative, and the patient refused fibroscopy. P. jirovecii was found via PCR in sputum collected on November 10, but the result of the test was missed. Improvement occurred after taking antibacterials, and prophylactic aerosols of pentamidine were reinstated, but 4 weeks later, the patient was hospitalized for febrile dyspnea. A BAL documented full-blown PCP with positive microscopy and PCR. She died from respiratory failure. We retrospectively determined from a sputum sample that the $P$. jirovecii genotype was pure Gt2.

Although Patients 4 and 9 share the rare Gt3 genotype, they never met.

\section{DISCUSSION}

We report here the documentation of interhuman transmission of $P$. jirovecii, which was between 4 allogeneic HSCT recipients. We used $P$. jirovecii genotyping and analysis of the transmission path in the hospital, which revealed that transmission probably occurred in the daycare center (Figure 2) where the patients repeatedly met for post-transplant follow-up. Contacts between the four Gt2-patients and the concomitance of PCP infection in Patient 2 and P. jirovecii detection in Patient 5 make it plausible that either Patient 2 or Patient 5 could have been the first transmitter. The Gt2 transmission could have started from the first PCP case (Patient 2), who transmitted the disease to Patients 5 and 10, either of whom could have contaminated Patient 7. Alternatively, because Patient 5 was retrospectively identified as a Gt2 carrier, she could have been the first transmitter.

This transmission likely occurred because of the absence of effective PCP prophylaxis. Indeed, out of 30 PCP outbreaks

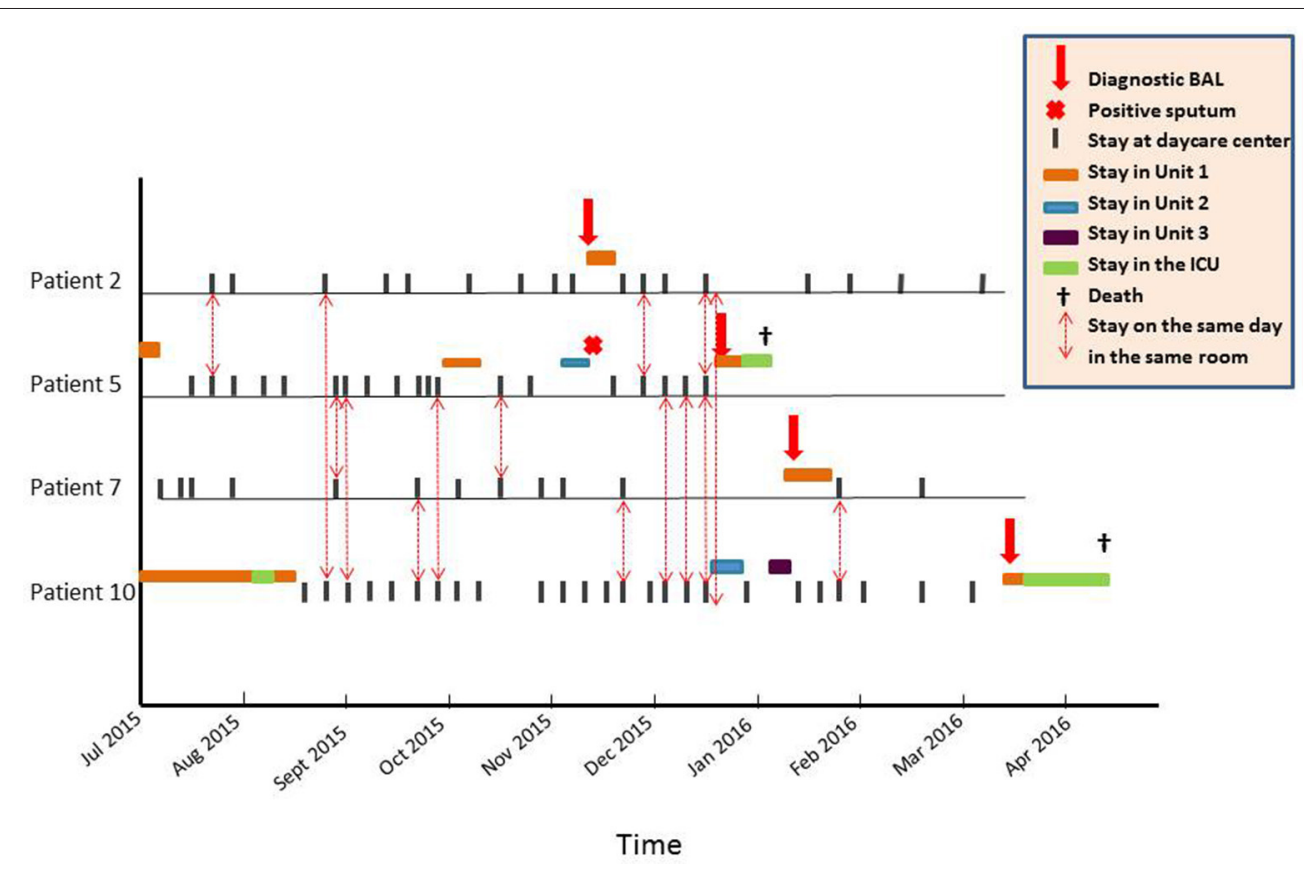

FIGURE 2 | Transmission map of the 4 patients infected with genotype 2 Pneumocystis jirovecii in the hematology ward, showing that any meeting between two Gt2 patients must have been in the daycare center because no 2 patients were concomitantly hospitalized in the same unit of the ward. Patient 2 met Patients 5 and 10 once each before developing PCP and twice each after developing PCP while under treatment followed by secondary prophylaxis, including once when both were met on the same day. Patient 2 never met Patient 7 . Patient 5, the only one to have met all 3 others at least once, met Patient 2 three times, Patient 7 twice, and Patient 10 five times. Patient 7 met Patient 5 twice and Patient 10 three times (twice before the diagnosis of PCP, and once thereafter). Patient 7 never met Patient 2. Patient 10 met Patient 2 twice and Patient 5 five times, including one occurrence where both were met on the same day. Patient 10 met Patient 7 three times. 
reviewed by Yiannakis et al., in 2016, 25 (83\%) were reported in kidney transplant recipients, and most were not receiving PCP prophylaxis (Yiannakis and Boswell, 2016). In the hematology ward, there are usually written procedures for PCP prophylaxis, at least for the most at-risk patients (i.e., allogeneic HSCT recipients, acute lymphoblastic leukemia and lymphoma patients). The first-choice prophylactic regimen in atrisk patients is TMP-SMX (Stern et al., 2014; Maertens et al., 2016). However, this can be associated with side effects, poor compliance, or omission. Although the staff complies with our policies, none of the 12 patients included in this study was receiving TMP-SMX at the time of PCP diagnosis. Because all four Gt2 patients were allogeneic HSCT recipients, they might have been at higher risk because of their repetitive, often weekly, appointments at the daycare center in spite of being a minority of our study population.

Until now, only two PCP outbreaks have been reported in hematology wards. In one, five diagnoses were reported within 6 months in the same unit but without molecular investigation (Ong and Jones, 1998). In the other, nested amplification, cloning, and sequencing of ITS showed a common ITS sequence type between two of the eight hematology study patients who shared the same room (Helweg-Larsen et al., 1998). However, the genotype was also the most common found in Denmark, where the study was performed. Therefore, the authors concluded that "person-to-person transmission may occur but it may be a relatively infrequent event" (Helweg-Larsen et al., 1998). On the other hand, a 2013 Belgian survey including 21 patients with $P$. jirovecii based on PCR of BAL fluid in the same hospital with six hematology patients failed to find transmission of the same $P$. jirovecii genotype using multilocus sequence typing (Depypere et al., 2016). In our series, nosocomial transmission in the daycare center between four of the 12 patients is amply supported. First, these patients shared a unique $P$. jirovecii genotype that had never been observed in our experience on more than 300 $P$. jirovecii strains across Europe (Gits-Muselli et al., 2015; Alanio et al., 2016a) while 70-92\% of PCP cases harbor mixtures of genotypes (Gits-Muselli et al., 2015; Alanio et al., 2016b; Depypere et al., 2016). Second, they had multiple opportunities to meet in the daycare center where they repetitively stayed several hours in the same, confined, waiting room. Finally, all were deeply immunosuppressed, and three were receiving no PCP prophylaxis.

Sharing the same genotype does not allow determination of the index case. Indeed, we do not know when transmission eventually occurred, but because a single pure genotype (Gt2) was found in four patients, a recent acquisition in these patients is suggested. Whether the transmission of Gt2 was direct, interhuman, the result of close contact, or was indirect through the environment can also not be determined. Healthcare workers could be the culprits, as suggested in other studies (Vargas et al., 2000; Miller et al., 2001; Valade et al., 2015), but this was not explored. Similarly, 16 outbreaks in kidney transplant patients have been documented using various molecular methods, and a single or predominant strain was found in 13 of them, supporting interhuman transmission either from patient to patient, via healthcare workers, or through the environment (Yiannakis and
Boswell, 2016). However, in none of these reports could all cases be ascribed to the same genotype, suggesting that both mechanisms of infection-reactivation and recent interhuman transmission-probably occurred concomitantly, whether or not in the setting of an outbreak. Multiple $P$. jirovecii genotypes could circulate at the same time, and from various people. As genotype mixtures are common in PCP patients (Gits-Muselli et al., 2015; Alanio et al., 2016b; Depypere et al., 2016), this precludes any conclusion about which genotype was initially present. Only monitoring of patients over a long period of time could elucidate this fact.

While it is important to note that transmission occurred between our study patients, one may consider that PCP was not confirmed in five of those included in this study. Indeed, Patients $2,7,8,9$, and 12 were microscopically negative using MGG or IFA staining and were serum $\beta$-D-glucan negative. However, all patients had clinical and imaging patterns consistent with PCP. Even if some patients are considered probable or putative cases, it does not change the possibility of $P$ jirovecii transmission among them. We do not know whether there is a minimal fungal load to contaminate another patient. It could be hypothesized that any cough generated by another bronchopulmonary infection, especially a respiratory viral infection, could contribute to increased transmission of $P$. jirovecii, whatever the fungal load. Therefore, our study confirms the interest of adding a $P$. jirovecii qPCR on respiratory samples of hematology patients with pulmonary symptoms to determine whether they are possible transmitters (Guigue et al., 2015).

To determine whether patients with low fungal loads are the source of infection or participate in the transmission chain is hampered by the inability to genotype when facing a low fungal burden. The qPCR used for $P$. jirovecii detection in respiratory samples targets a multicopy gene (mtLSUrRNA) to improve test sensitivity (Alanio et al., 2011; Botterel et al., 2012), whereas the alleles tested for genotyping are located on single-copy genes (Gits-Muselli et al., 2015). Therefore, other epidemiological links or transmission between patients harboring low fungal loads could have been missed. A typing system based on the investigation of mitochondrial markers (mainly by sequencing mtLSU or cytb genes) for genotyping would lead to increased sensitivity, but the reliability of this method is disputable because mitochondrial heteroplasmy and recombination could occur (Valero et al., 2016). Furthermore, the plasticity and mutation rates is known to differ between the mitochondrial genome and the nuclear genome. This is why mitochondrial genes have not been retained in the multilocus sequence typing approach for Candida albicans, for instance (Odds and Jacobsen, 2008).

In conclusion, this study illustrates two important features: (1) nosocomial transmission of $P$. jirovecii is possible between infected patients within the hematology ward, as illustrated in our daycare center; and (2) TMP-SMX, the optimal PCP prophylaxis (Maertens et al., 2016) is important, and its absence increases the susceptibility of at-risk patients during an outbreak. This clearly supports a strong, specific recommendation for isolation and respiratory precautions with PCP, and more generally, encourages reinforcing control policies when pulmonary symptoms are seen in the hematology ward. 
This recommendation was, up to now, not evidence-based for PCP in hematology (Siegel et al., 2007; Maertens et al., 2016). Thus, hospitalized PCP patients should be in singleoccupancy rooms and wear at least a surgical mask to prevent transmission when meeting other patients in confined waiting rooms, such as a daycare center. Wearing a mask could have prevented the onset of this outbreak. Our data support that these recommendations be extended to any PCP-at-risk patient with febrile pneumonia and the presence of $P$. jirovecii, detected using qPCR on respiratory specimens. Wearing a mask in the hospital for any hematology patient without respiratory symptom should logically decrease the transmission of any pathogen transmitted through air, and subsequently decrease the incidence of other respiratory diseases, especially during the fall-winter season. The efficacy and acceptability of such measure should, however, be carefully assessed.

\section{REFERENCES}

Alanio, A., Desoubeaux, G., Sarfati, C., Hamane, S., Bergeron, A., Azoulay, E., et al. (2011). Real-time PCR assay-based strategy for differentiation between active Pneumocystis jirovecii pneumonia and colonization in immunocompromised patients. Clin. Microbiol. Infect. 17, 1531-1537. doi: 10.1111/j.1469-0691.2010.03400.x

Alanio, A., Gits-Muselli, M., Calderon, E., Di Cave, D., Dupont, D., Hamprecht, A., et al. (2016a). "European study on Pneumocystis jirovecii short tandem repeats genotyping reveals wide population diversity with geographic specificities," in European Congress on Clinical Microbiology and Infectious Diseases (Amsterdam). doi: 10.1016/j.mycmed.2016.04.017

Alanio, A., Gits-Muselli, M., Mercier-Delarue, S., Dromer, F., and Bretagne, S. (2016b). Diversity of Pneumocystis jirovecii during infection revealed by ultradeep pyrosequencing. Front. Microbiol. 7:733. doi: 10.3389/fmicb.2016.00733

Alanio, A., Hauser, P. M., Lagrou, K., Melchers, W. J., Helweg-Larsen, J., Matos, O., et al. (2016c). ECIL guidelines for the diagnosis of Pneumocystis jirovecii pneumonia in patients with haematological malignancies and stem cell transplant recipients. J. Antimicrob. Chemother. 71, 2386-2396. doi: $10.1093 / \mathrm{jac} / \mathrm{dkw} 156$

Alanio, A., Morio, F., Gits-Muselli, M., Desnos-Ollivier, M., Maitte, C., and Bretagne, S. (2014). "Short tandem repeat genotyping for $P$. jirovecii is more sensitive to mixed genotype than MLST," in International Workshop on Opportunistic Protists (IWOP) (Sevilla).

Botterel, F., Cabaret, O., Foulet, F., Cordonnier, C., Costa, J. M., and Bretagne, S. (2012). Clinical significance of quantifying Pneumocystis jirovecii DNA by using real-time PCR in bronchoalveolar lavage fluid from immunocompromised patients. J. Clin. Microbiol. 50, 227-231. doi: 10.1128/JCM.06036-11

Choukri, F., Menotti, J., Sarfati, C., Lucet, J. C., Nevez, G., Garin, Y. J. F., et al. (2010). Quantification and spread of Pneumocystis jirovecii in the surrounding air of patients with Pneumocystis pneumonia. Clin. Infect. Dis. 51, 259-265. doi: $10.1086 / 653933$

Cordonnier, C., Cesaro, S., Maschmeyer, G., Einsele, H., Donnelly, J. P., Alanio, A., et al. (2016). Pneumocystis jirovecii pneumonia: still a concern in patients with haematological malignancies and stem cell transplant recipients. J. Antimicrob. Chemother. 71, 2379-2385. doi: 10.1093/jac/dkw155

Damiani, C., Choukri, F., Le Gal, S., Menotti, J., Sarfati, C., Nevez, G., et al. (2012). Possible nosocomial transmission of Pneumocystis jirovecii. Emerg. Infect. Dis. 18, 877-878. doi: 10.3201/eid1805.111432

de Boer, M. G., de Fijter, J. W., and Kroon, F. P. (2011). Outbreaks and clustering of Pneumocystis pneumonia in kidney transplant recipients: a systematic review. Med. Mycol. 49, 673-680. doi: 10.3109/13693786.2011.571294

Depypere, M., Saegeman, V., and Lagrou, K. (2016). Typing of Pneumocystis jirovecii by multilocus sequencing: evidence of outbreak? Eur. J. Clin. Microbiol. Infect. Dis. 35, 911-916. doi: 10.1007/s10096-016-2615-y

\section{AUTHOR CONTRIBUTIONS}

$\mathrm{CR}, \mathrm{AA}$, and CC conceived and designed the study. CR, AA, MG, GL, FS, ML, FlB, RR, CP, AT, and SM provided study materials or patients. CR, AA, GL, FrB, and CA collected and assembled data. $\mathrm{CR}, \mathrm{AA}, \mathrm{CC}$, and SB analyzed and interpreted data. CR, AA, SB, and $\mathrm{CC}$ drafted the manuscript. All authors approved the final version.

\section{ACKNOWLEDGMENTS}

The authors are grateful to the technical staff of the laboratory of mycology at Henri Mondor and Saint-Louis hospitals. They are also grateful to the physicians of the hematology department and of the pneumology and intensive care units of Henri Mondor hospital.

Desoubeaux, G., Dominique, M., Morio, F., Thepault, R. A., Franck-Martel, C., Tellier, A. C., et al. (2016). Epidemiological outbreaks of Pneumocystis jirovecii pneumonia are not limited to kidney transplant recipients: genotyping confirms common source of transmission in a liver transplantation unit. J. Clin. Microbiol. 54, 1314-1320. doi: 10.1128/JCM.00133-16

Gajdusek, D. C. (1957). Pneumocystis carinii; etiologic agent of interstitial plasma cell pneumonia of premature and young infants. Pediatrics 19, 543-565.

Gigliotti, F., and Wright, T. W. (2012). Pneumocystis: where does it live? PLoS Pathog. 8:e1003025. doi: 10.1371/journal.ppat.1003025

Gits-Muselli, M., Peraldi, M. N., de Castro, N., Delcey, V., Menotti, J., Guigue, N., et al. (2015). New short tandem repeat-based molecular typing method for Pneumocystis jirovecii reveals intrahospital transmission between patients from different wards. PLoS ONE 10:e0125763. doi: 10.1371/journal.pone.01 25763

Guigue, N., Alanio, A., Menotti, J., Castro, N. D., Hamane, S., Peyrony, O., et al. (2015). Utility of adding Pneumocystis jirovecii DNA detection in nasopharyngeal aspirates in immunocompromised adult patients with febrile pneumonia. Med. Mycol. 53, 241-247. doi: 10.1093/mmy/myu087

Helweg-Larsen, J., Tsolaki, A. G., Miller, R. F., Lundgren, B., and Wakefield, A. E. (1998). Clusters of Pneumocystis carinii pneumonia: analysis of person-toperson transmission by genotyping. QJM 91, 813-820. doi: 10.1093/qjmed/ 91.12 .813

Maertens, J., Cesaro, S., Maschmeyer, G., Einsele, H., Donnelly, J. P., Alanio, A., et al. (2016). ECIL guidelines for preventing Pneumocystis jirovecii pneumonia in patients with haematological malignancies and stem cell transplant recipients. J. Antimicrob. Chemother. 71, 2397-2404. doi: 10.1093/jac/ dkw157

Maschmeyer, G., Helweg-Larsen, J., Pagano, L., Robin, C., Cordonnier, C., and Schellongowski, P. (2016). ECIL guidelines for treatment of Pneumocystis jirovecii pneumonia in non-HIV-infected haematology patients. J. Antimicrob. Chemother. 71, 2405-2413. doi: 10.1093/jac/dkw158

Miller, R. F., Ambrose, H. E., and Wakefield, A. E. (2001). Pneumocystis carinii f. sp. hominis DNA in immunocompetent health care workers in contact with patients with P. carinii pneumonia. J. Clin. Microbiol. 39, 3877-3882. doi: 10.1128/JCM.39.11.3877-3882.2001

Morris, A., and Norris, K. (2012). Colonization by Pneumocystis jirovecii and its role in disease. Clin. Microb. Rev. 25, 297-317. doi: 10.1128/CMR.00 013-12

Odds, F. C., and Jacobsen, M. D. (2008). Multilocus sequence typing of pathogenic Candida species. Eukaryot Cell 7, 1075-1084. doi: 10.1128/EC.00062-08

Ong, Y. L., and Jones, F. G. (1998). A cluster of suspected Pneumocystis carinii pneumonia following intensive chemotherapy in a Belfast haematology unit. Ulster Med. J. 67, 104-109.

Parobek, C. M., Jiang, L. Y., Patel, J. C., Alvarez-Martinez, M. J., Miro, J. M., Worodria, W., et al. (2014). Multilocus microsatellite genotyping array 
for investigation of genetic epidemiology of Pneumocystis jirovecii. J. Clin. Microbiol. 52, 1391-1399. doi: 10.1128/JCM.02531-13

Siegel, J. D., Rhinehart, E., Jackson, M., Chiarello, L., and Health Care Infection Control Practices Advisory Committee. (2007). Guideline for isolation precautions: preventing transmission of infectious agents in health care settings. Am. J. Infect. Control 35(10 Suppl. 2), S65-S164. doi: 10.1016/j.ajic. 2007.10.007

Stern, A., Green, H., Paul, M., Vidal, L., and Leibovici, L. (2014). Prophylaxis for Pneumocystis pneumonia (PCP) in non-HIV immunocompromised patients. Cochrane Database Syst. Rev. 10:Cd005590. doi: 10.1002/14651858.CD005590.pub3

Thomas, C. F. Jr. and Limper, A. H. (2004). Pneumocystis pneumonia. N. Engl. J. Med. 350, 2487-2498. doi: 10.1056/NEJMra032588

Valade, S., Azoulay, E., Damiani, C., Derouin, F., Totet, A., and Menotti, J. (2015). Pneumocystis jirovecii airborne transmission between critically ill patients and health care workers. Intensive Care Med. 41, 1716-1718. doi: 10.1007/s00134-015-3835-9

Valero, C., Buitrago, M. J., Gits-Muselli, M., Benazra, M., Sturny-Leclere, A., Hamane, S., et al. (2016). Copy number variation of mitochondrial DNA genes in Pneumocystis jirovecii according to the fungal load in BAL specimens. Front. Microbiol. 7:1413. doi: 10.3389/fmicb.2016.01413
Vargas, S. L., Ponce, C. A., Gigliotti, F., Ulloa, A. V., Prieto, S., Munoz, M. P., et al. (2000). Transmission of Pneumocystis carinii DNA from a patient with P. carinii pneumonia to immunocompetent contact health care workers. J. Clin. Microbiol. 38, 1536-1538.

Yiannakis, E. P., and Boswell, T. C. (2016). Systematic review of outbreaks of Pneumocystis jirovecii pneumonia: evidence that $P$. jirovecii is a transmissible organism and the implications for healthcare infection control. J. Hosp. Infect. 93, 1-8. doi: 10.1016/j.jhin.2016.01.018

Conflict of Interest Statement: The authors declare that the research was conducted in the absence of any commercial or financial relationships that could be construed as a potential conflict of interest.

Copyright (®) 2017 Robin, Alanio, Gits-Muselli, la Martire, Schlemmer, Botterel, Angebault, Leclerc, Beckerich, Redjoul, Pautas, Toma, Maury, Bretagne and Cordonnier. This is an open-access article distributed under the terms of the Creative Commons Attribution License (CC BY). The use, distribution or reproduction in other forums is permitted, provided the original author(s) or licensor are credited and that the original publication in this journal is cited, in accordance with accepted academic practice. No use, distribution or reproduction is permitted which does not comply with these terms. 